SPATIAL COGNITION AND COMPUTATION, 7(2), 213-226

Copyright @ $\odot$ 2007, Lawrence Erlbaum Associates, Inc.

\title{
Spatial Reorientation: Effects of Verbal and Spatial Shadowing
}

\author{
Almut Hupbach, ${ }^{1}$ Oliver Hardt, ${ }^{2}$ Lynn Nadel, ${ }^{3}$ \\ and Veronique D. Bohbot ${ }^{1,2}$ \\ ${ }^{1}$ Douglas Hospital Research Centre, Verdun, QC, Canada \\ ${ }^{2}$ McGill University, Montreal, QC, Canada \\ ${ }^{3}$ The University of Arizona, Tucson, AZ, USA
}

\begin{abstract}
Spatially disoriented adults flexibly conjoin geometric information (macroscopic shape) and nongeometric information (e.g., the color of a wall) to re-establish their bearings. It has been proposed that non-geometric information is incorporated into a geometric frame of reference through the use of spatial language. Support for this assumption comes from dual-task studies in which participants failed to use non-geometric features when they shadowed a verbal message concurrent to the reorientation task. These studies were performed in small rectangular spaces. In contrast, our study was performed in a large square room. Experiment 1 showed that verbal shadowing did not disrupt non-geometric feature use in this environment. In Experiment 2, we found that a spatial shadowing task that required the encoding of frequently changing spatial directions impaired reorientation behavior. Our study shows that nongeometric information can be used for reorientation without recourse to linguistic processes, and suggests that the use of non-geometric features is dependent upon a spatial coding process.
\end{abstract}

Keywords: spatial orientation, spatial memory, verbal shadowing, geometric module

\section{INTRODUCTION}

Mobile organisms use a variety of cognitive mechanisms to orient in space. Path integration (or dead reckoning), cue and response learning, and cognitive mapping can all contribute to locating food, avoiding dangerous places,

Correspondence concerning this article should be sent to Dr. Almut Hupbach, Department of Psychology, University of Arizona, Tucson, AZ 85721, USA; email: ahupbach@email.arizona.edu 
relocating the nest etc. Under normal conditions, these mechanisms work in concert, correcting and minimizing errors resulting from the use of each mechanism alone. In path integration, for instance, an animal computes a vector pointing towards its starting location by continuously updating its linear and directional displacement from that starting location while moving around. Path integration is prone to the slow accumulation of error, and animals use external cues to periodically update their path with respect to their target, for example when they return to their starting position. But what happens when an animal loses its sense of direction and its bearings? What processes and cues do organisms use to reorient themselves?

Cheng (1986) found that disoriented rats relied solely upon geometric features of the environment when trying to relocate a previously visited food source in a rectangular box despite the presence of various salient nongeometric features such as odor, texture and color. The shape of a rectangle provides ambiguous information about an object's location because the two diagonally opposite corners share the same geometric information. Because disoriented rats failed to use available non-geometric information to resolve this ambiguity, Cheng concluded that rats represent space in a modular fashion, relying on a "geometric module," a featureless metric frame that "records the geometric relations in the arrangement of surfaces as surfaces" (Cheng, 1986, p. 175; for the concept of modules, see Fodor, 1983). By this account, information about other features of the environment, such as odor, color, etc. is processed in separate modules, and does not penetrate the geometric module. Attempts to replicate this finding in other species have yielded mixed results (for a review, see Cheng \& Newcombe, 2005).

Hermer and Spelke $(1994,1996)$ proposed that the geometric module is a phylogenetically old spatial system shared by a variety of species including humans. They showed that disoriented children also rely upon geometric information and are incapable of integrating salient non-geometric features such as a colored wall when retrieving an object hidden prior to the disorientation procedure. Human adults, however, overcome the constraints of the geometric module and are able to flexibly integrate nongeometric features in solving the disorientation problem. Hermer-Vazquez and colleagues (Hermer-Vazquez, Spelke, \& Katsnelson, 1999; Hermer-Vazquez, Moffet, \& Munkholm, 2001) hypothesized that language is the medium by which information from different encapsulated systems can be combined. This hypothesis is based on two empirical observations: First, young children behave like Cheng's rats when trying to relocate a hidden object in a small rectangular chamber containing nongeometric features (Hermer \& Spelke, 1994, 1996). Around 5 years of age, when most children are able to correctly apply the terms "left" and "right," they start to use these nongeometric features and commit fewer rotational errors, i.e., incorrect searches at the diagonally opposite corner. Additionally, the successful production of the terms "left" and "right" correlates with the use of nongeometric information in the reorientation task (Hermer-Vazquez et al., 2001). Second, when adults were asked to 
continuously repeat an auditory recording (verbal shadowing) while performing the reorientation task, they failed to incorporate nongeometric features and showed similar search patterns as young children and rats (Hermer-Vazquez et al., 1999). In contrast, a concurrent rhythm-clapping task did not have the same disrupting effect.

There are, however, several issues that complicate the interpretation of these findings. While the study with children (Hermer-Vazquez et al., 2001) revealed a correlation between the production of the spatial terms "left" and "right" and the successful use of nongeometric features, it is unclear whether there is a causal relationship between the development of spatial language and integration of non-geometric information or whether it is a spurious correlation, i.e. spatial language production and nongeometric feature use are unrelated and simply emerge around the same age. Indeed, Hupbach and Nadel (2005) found that when age was statistically controlled for, the correlation between spatial language production and successful reorientation was reduced and no longer significant.

Newcombe (2005) argued that verbal shadowing could engage brain structures that are also needed for feature integration. For instance, both verbal encoding and categorical coding of features are accompanied by leftlateralized activation of the medial-temporal lobe. Hence activation of similar neuronal circuits could have caused the verbal task to interfere with feature use.

Another critical aspect of Hermer-Vazquez et al.'s $(1999,2001)$ studies is that they were performed in small rectangular spaces $(1.22 \times 1.83 \mathrm{~m}, 1.68 \times$ $2.32 \mathrm{~m}$ ). Participants were embedded in the space (in comparison to viewing it from outside), but given the size, could hardly move around. Studies by Learmonth and colleagues (Learmonth, Newcombe, \& Huttenlocher, 2001; Learmonth, Nadel, \& Newcombe, 2002) and Hupbach and Nadel (2005) have shown that room size, and probably the ability to freely move within a space, critically influence reorientation behavior. Young children can combine geometric and non-geometric information to reorient in larger spaces. ${ }^{1}$ This could imply that a verbal process is not critical for feature use in large spaces. This is what we sought to test.

In Experiment 1, we asked whether a verbal shadowing task would interfere with feature use in a large space in which participants could freely move and walk around. To further simplify the task situation, we used a square

\footnotetext{
${ }^{1}$ In the present article, we refer to small spaces as spaces with a floor space less than $1.7 \times 2.4 \mathrm{~m}$ (Hermer-Vazquez et al., 1999, 2001). Spaces of this size make it hard for participants to move around. We refer to larger spaces as spaces with a floor plan varying between $2.4 \times 3.7 \mathrm{~m}$ (Learmonth et al., 2001, 2002; Ratliff \& Newcombe, submitted) and $5.0 \times 5.0 \mathrm{~m}$ (present study). This classification is solely based on the various room sizes that have been used in empirical studies and is not at all meant to be a classification with external validity. For a topology of geographic space based on manipulability, locomotion, and size, see Freundschuh and Egenhofer (1997).
} 
instead of a rectangular room. In a rectangular room, two corners share the same geometric information (e.g., located on the left side of the long and the right side of the short wall), and participants using just geometric information have a $50 \%$ chance of finding the correct corner. In a square room, all four corners share the same geometrical information and participants using just geometric information have only a $25 \%$ chance of finding the actual hiding place.

\section{EXPERIMENT 1}

\section{Methods}

Design \& Participants. A within-subject design, modeled after Experiment 1 of Hermer-Vazquez et al.'s (1999) study was used. During the first eight trials, one wall of the test room provided a landmark. Participants were asked to find a hidden object in one of the four corners of the room. On the first four trials participants performed a secondary task during which they continuously repeated a verbal message. On trials 5-8, they did not perform this secondary task. After the landmark condition, a no-landmark condition (trials: 9-12) followed in which all four walls were colored a uniform yellow and participants did not perform a secondary task. This condition served as a control to show that the room itself could not provide any information about the target corner.

Thirteen participants ( 7 females, 6 males) took part in the experiment. They were undergraduate students and employees who were affiliated with the Douglas Hospital Research Center or McGill University. Two females were excluded from the analyses, because they reported keeping track of their position by counting turns during the disorientation procedure, i.e., they did not allow themselves to become disoriented as was also revealed by their nearly perfect search patterns in the all-yellow room.

Material. The experimental apparatus consisted of a $5.0 \times 5.0 \mathrm{~m}$ square room. The walls and the ceiling were painted black. The floor was covered with a featureless gray carpet. Two doors were located on each wall at an equal distance from the left and right corners. Lighting came from several small lamps symmetrically distributed across the ceiling. A large screen, displaying a single color, was located in the center of each wall. In the no-landmark condition, all screens were yellow. In the landmark condition, one screen was blue. Eight speakers, also symmetrically distributed, were mounted to the ceiling. A small featureless coffee cup was placed upside down on the floor in each corner and served as the hiding location. A small sugar packet served as the hidden object. The message that was used for the verbal shadowing task was a fairy tale (human-read audio book). All eight speakers simultaneously broadcast the message. 
Procedure. Participants were brought into the middle of the experimental room, where they remained throughout the study. They started by practicing the verbal shadowing task. Participants were asked to continuously repeat the spoken narrative syllable by syllable and not to wait for words or sentences to be completed. After they were able to continuously shadow for about two minutes without pausing for more than two seconds, instructions for the reorientation task were given. As in Hermer-Vazquez et al.'s (1999) study, participants received the following rather unspecific instruction: "You will see something happening during the experiment that you should try to notice. You will be asked about what you saw."

Additionally, participants were told that they would be asked to spin themselves around and that they should allow themselves to get disoriented rather than attempting to maintain their sense of orientation. A trial started with having the participants shadow the verbal message. After 1 minute, the experimenter directed the participants' attention to the sugar packet and hid it under one of the cups. Then, participants were asked to close their eyes and turn themselves around. After about 5 to 7 turns, the experimenter asked participants to change their turning direction. After an additional 5 to 7 turns, the experimenter stopped participants by touching their shoulders. For every trial, they were stopped at a predefined facing direction such that each wall was faced once in all three conditions. The sequence of facing directions was randomized for each participant. When the experimenter stopped the participant, s/he always stood behind him/her facing the same direction as the participant. On each trial, participants were allowed only one attempt to retrieve the sugar packet by pointing to one of the cups. The experimenter made note of the location and retrieved the sugar packet while participants were watching. Immediately after that, the next trial started. Across the four trials in each condition all four possible hiding locations were used once, and the order of locations was randomized. The four trials of the verbal shadowing condition were followed by a short break during which the sound was turned off. Then, the four trials of the no-shadowing landmark condition were administered. After another short break during which the blue screen was changed to yellow, the four trials of the no-landmark condition were administered.

\section{Results}

The mean number of searches in each corner averaged over the four trials served as the dependent variable (see Figure 1, top row).

\section{Landmark Conditions: Verbal Shadowing vs. No Shadowing}

During the first 8 trials, one of the walls had a landmark (blue screen), and on the first 4 of these trials participants shadowed the verbal message. 


\section{EXPERIMENT 1}

Landmark Condition:

Verbal Shadowing

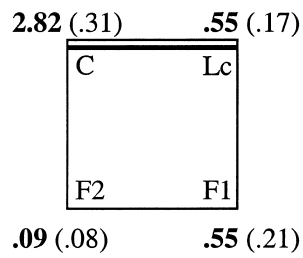

\section{EXPERIMENT 2}

Landmark Condition:

Spatial Shadowing

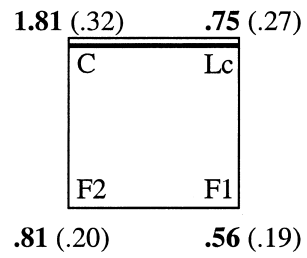

Landmark Condition:

No Shadowing

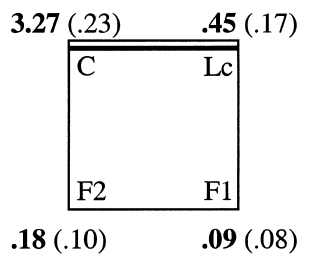

Landmark Condition: No Shadowing



No-landmark

condition

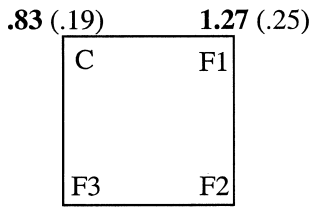

$1.27(.23) \quad .64(.13)$

Figure 1. Mean number of searches (and standard errors) summed over 4 trials for Experiment 1 (verbal shadowing) and 2 (spatial shadowing). Note: For the purpose of simplicity, the upper left corner is displayed as the hiding location. In the experiment, the hiding location varied from trial to trial such that each hiding location was used for each participant and condition (shadowing, no-shadowing, control) once.

Behavioral responses were characterized in 3 ways: (1) correct corner $(C)$; (2) incorrect corner in a correct place relative to the landmark, but mirror reversed (landmark-congruent, $L c$ ); (3) incorrect corner relative to the landmark (false-1, F1, and false-2, F2).

First, we compared landmark-congruent searches ( $\mathrm{C}$ and $\mathrm{Lc}$ ) to landmarkincongruent searches ( $\mathrm{F} 1$ and $\mathrm{F} 2$ ) with a 2 (landmark-congruent vs. landmarkincongruent $) \times 2$ (verbal shadowing vs. no shadowing) repeated-measures ANOVA. The ANOVA revealed only a significant main effect of landmarkcongruency $[F(1,10)=96.33, M S E=1.09, p<.01$; all other effects were nonsignificant, $F \leq 1.46$ ]. Participants searched significantly more often in the landmark congruent than in the landmark incongruent corners. Performance under the shadowing condition did not differ from that of the non-shadowing condition.

Second, we compared searches at the two landmark-congruent corners with a 2 (correct vs. adjacent landmark-congruent) $\times 2$ (verbal shadowing vs. no shadowing) repeated-measures ANOVA. We found a main effect of 
corner $[F(1,10)=34.39, M S E=2.07, p<.01$; all other effects were nonsignificant $F \leq 1.32$ ]. The correct corner was searched significantly more often than the adjacent, but incorrect, landmark-congruent corner. Performance under the shadowing condition did not differ from that of the non-shadowing condition.

\section{No-Landmark Condition}

In the no-landmark condition, corners were searched randomly $[F(3,56)=$ $1.30, M S E=.89, p=.29]$ thus confirming that the apparatus itself did not provide any hints about the target location.

\section{Discussion}

Participants used the distinctively colored wall in both the verbal shadowing and the no-shadowing condition: landmark-congruent corners were searched more often than landmark-incongruent corners. Additionally, participants mostly searched at the correct corner and made few incorrect choices of the adjacent-landmark congruent corner. Most importantly, there was no difference between the shadowing and the no-shadowing condition. Participants used the unique color of the blue screen and sense information (e.g., "The sugar is hidden on the left side of the blue wall") in both conditions. The finding that verbal shadowing did not interfere with successful object retrieval is in direct contrast to Hermer-Vazquez et al.'s (1999) finding and argues against the view that a verbal coding process is responsible for landmark use, at least in a large square room.

The numerical difference between correct searches in the verbal shadowing and no-shadowing condition could reflect an unspecific training effect: All participants started with the shadowing condition and received a rather unspecific general instruction (“... something will happen that you should try to notice"). They might have needed some time to realize that the task at hand involved object retrieval. This interpretation finds support in recent work by Ratliff and Newcombe (submitted). They studied verbal shadowing effects in a small rectangular space and found significant differences between an unspecific instruction and an explicit explanation of the object retrieval task that was paired with practice trials. No effects of verbal shadowing were found for the explicit condition, but verbal shadowing impaired object retrieval in the unspecific condition.

Our results could have differed from those reported by Hermer-Vazquez et al. (1999) either because we used a larger room or because we used a square room in which geometry did not provide hints about the target corner. We would argue that room size is responsible for the discrepant results, because Ratliff and Newcombe (submitted) found verbal shadowing effects in 
the unspecific instruction condition only in the small, but not in a large rectangular space. Only in larger spaces do landmarks have the quality of distal cues. In contrast, in small spaces, which do not allow for much movement, all landmarks are proximal in nature. Animal studies show that it is the distal cues that dominantly guide reorientation behavior (e.g., Zugaro et al., 2004). Therefore, it not surprising that landmarks are used very differently in small and larger spaces (for further discussion of why room size matters, see Nadel \& Hupbach, 2005; Newcombe, 2006).

Ratliff and Newcombe's (submitted) and our findings show that landmark integration in a larger space does not appear to rely upon a linguistic coding process. It is possible that the verbal shadowing task used by HermerVazquez et al. (1999) disrupted the ability to use featural landmarks not (or not only) by interfering with a linguistic encoding process, but by interfering with a nonlinguistic spatial coding system (Newcombe, 2005). If so, a shadowing task that involves spatial processing should interfere with the use of landmarks. This was studied in Experiment 2.

\section{EXPERIMENT 2}

In Experiment 2, we asked whether a shadowing task that required spatial location coding would interfere with landmark use in a large square room. A short tone was broadcasted by one of the eight speakers, and participants were asked to point towards the speaker that currently displayed the sound. This task was similar to the verbal shadowing task used in Experiment 1 in that the to-be-shadowed information was delivered by the speakers and that the participants remained in a fixed position within the center of the room. In this case, however, the to-be-shadowed information and the response were both nonverbal in nature. Since the encoding of this information relies upon a spatial rather than verbal coding process, we expected to find reorientation difficulties in the spatial shadowing condition.

\section{Methods}

Design \& Participants. The design was identical to Experiment 1 with the difference that verbal shadowing was replaced by spatial shadowing. On trials 1 to 4 , research participants underwent reorientation simultaneously with spatial shadowing. This was followed by 4 trials of reorientation with a single landmark and 4 trials without landmark.

Twenty-one participants (11 females, 10 males) took part in the experiment. They consisted of undergraduate students and employees who were affiliated with the Douglas Hospital Research Center or McGill University. Three females and two males were excluded from the analyses, because they reported that they did not allow themselves to get disoriented. Their lack 
of disorientation was also revealed by the nearly perfect searches in the allyellow room. Although the number of excluded participants seems high, it is not uncommon in reorientation experiments for adults to try to solve the task by engaging in strategies that counteract the disorientation procedure, such as keeping track of the number of rotational turns (cf. Hermer-Vazquez et al., 1999). Importantly, the strategies the excluded participants used were unrelated to the spatial or verbal coding process in question.

Material. The experiment took place in the same room and setup as Experiment 1 . The only difference concerned the spatial shadowing condition: The speakers broadcast beeping sounds for $1 \mathrm{sec}$, with only one of the speakers broadcasting at any given time. The speakers came on in a random order with a $1 \mathrm{~s}$ interval between tones.

Procedure. The procedure was identical to Experiment 1 except for the shadowing condition. Participants were asked to point towards the speaker that was currently broadcasting the tone. Participants were positioned in the center of the room and were free to choose their facing direction throughout the experiment. Before starting the object retrieval task, participants shadowed until they were able to point towards the correct speakers without error for 5 consecutive trials. A trial began with the participants pointing to the beeping sounds and watching the experimenter place the sugar packet in the cup. This was followed by the disorientation phase and choice, while performing the spatial shadowing task concurrently. Following the 4 spatial shadowing and reorientation trials, participants underwent 4 reorientation trials with and 4 reorientation trials without a landmark as described in Experiment 1.

\section{Results}

The mean number of searches in each corner averaged over the four trials served as the dependent variable (see Figure 1, bottom row). As in the previous experiment, behavioral responses were characterized in 3 ways: (1) correct corner $(C)$; $(2)$ incorrect corner in a correct place relative to the landmark, but mirror reversed $(L c)$; (3) incorrect corner relative to the landmark (F1 and F2).

\section{Landmark Conditions: Spatial Shadowing vs. No Shadowing}

First, we compared landmark-congruent searches ( $\mathrm{C}$ and $\mathrm{Lc})$ to landmarkincongruent searches (F1 and F2) with a 2 (landmark-congruent vs. landmarkincongruent) $\times 2$ (spatial shadowing vs. no shadowing) repeated-measures ANOVA. The ANOVA revealed a significant main effect of landmark-congruency $[F(1,15)=53.09, M S E=1.58, p<.01]$. Additionally, the interaction between the landmark-congruency and the shadowing condition was 
significant $[F(1,15)=27.23, M S E=.69, p<.01]$. Further analyses of the simple main effects revealed that participants searched landmark-congruent corners significantly more often than landmark-incongruent corners in both the spatial shadowing $[F(1,15)=6.44, M S E=1.81, p=.02]$ and the noshadowing condition $[F(1,15)=198.82, M S E=.46, p<.01]$. Additionally, participants searched landmark-congruent corners less often in the spatial shadowing condition than in the no-shadowing condition $[F(1,15)=27.23$, $M S E=.34, p<.01]$. For the landmark-incongruent corners, this effect was reversed, i.e., participants searched landmark-incongruent corners more often in the spatial shadowing condition than in the no-shadowing condition $[F(1,15)=27.23, M S E=.34, p<.01]$.

Second, we compared searches at the two landmark-congruent corners with a 2 (correct vs. adjacent landmark-congruent) $\times 2$ (spatial shadowing vs. no-shadowing) repeated-measures ANOVA. We found main effects of corner $[F(1,15)=27.31, M S E=3.00, p<.01]$, shadowing condition $[F(1,15)=$ $17.55, M S E=.17, p<.01]$ and a significant interaction between these factors $[F(1,15)=10.00, M S E=.90, p<.01]$. Further analyses of the simple main effects revealed that participants searched the correct corner significantly more often than the adjacent landmark-congruent corner in the no-shadowing condition $[F(1,15)=32.87, M S E=1.60, p<.01]$. In the spatial shadowing condition, this difference did not reach significance $[F(1,15)=3.93, M S E=2.30, p=.07]$. Importantly, participants searched the correct corner significantly less often in the spatial shadowing than in the no-shadowing condition $[F(1,15)=26.78, M S E=.51, p<.01]$. Search rates at the adjacent-landmark congruent corner did not differ between the two conditions $[F<1]$.

\section{No-Landmark Condition}

In the no-landmark condition, corners were searched randomly $[F(3,45)=$ $1.55, M S E=.56, p=.21]$ thus confirming that the apparatus itself did not provide hints to the target location.

\section{Discussion}

Experiment 2 showed that reorientation with respect to landmark and sense information was impaired when participants were concurrently engaged in a spatial shadowing task requiring encoding of spatial direction. In the spatial shadowing condition, participants relied less often on feature information, i.e., they searched landmark-congruent corners less than in the no-shadowing condition. Additionally, spatial shadowing made it difficult to differentiate between the correct and the adjacent landmark-congruent corner, i.e., the spatial shadowing task not only interfered with feature use, but also with the additional incorporation of sense information. Taken together, these findings 
support the view that the combined use of feature and sense information depends upon a spatial rather than verbal coding process. Ratliff and Newcombe (submitted) also report impairments caused by a spatial visualization task for a small rectangular space, but they did not find such an effect for a larger rectangular space. However, they used a different spatial task in which participants had to visualize letters and categorize intersecting points. This task differed from ours in a number of ways: In the visualization task, the response was verbal in nature and the to-be-evaluated information was imaginative. In our study, participants had to continuously track the source of a sound, requiring continuous large-scale direction coding, which might have made our task more disruptive.

\section{GENERAL DISCUSSION}

Hermer-Vazquez et al. $(1999,2001)$ proposed that reorientation is solely based on geometry by nonhuman species and human children because the incorporation of nongeometric features depends upon a linguistic process, specifically, the production of the terms left and right. This hypothesis is based on two types of studies, both of which were conducted in small rectangular enclosures: (1) correlational studies showing that children start to use nongeometric features at the same time as they start to successfully apply the terms left and right (Hermer-Vazquez et al., 2001), and (2) studies with human adults showing that they fail to use nongeometric features when they concurrently have to perform a verbal shadowing task (Hermer-Vazquez et al., 1999; Ratliff \& Newcombe, submitted).

Contrary to this assumption, the present study, using a large square room, shows that disoriented human adults re-established their orientation by relying on both nongeometric and geometric features even when linguistic processes were occupied by a secondary task. While linguistic processes might be helpful for reorientation purposes, they are certainly not necessary (cf. Ratliff \& Newcombe, submitted).

Recent studies with animals have also cast doubt on the geometric module hypothesis in general and the linguistic coding hypotheses in particular. While chicks (Vallortigara, Zanforlin, \& Pasti, 1990) use either only geometric or only nongeometric information depending on the specific task procedure, pigeons (Kelly, Spetch, \& Heth, 1998), fish (Sovrano, Bisazza, \& Vallortigara, 2002, 2003), and rhesus monkeys (Gouteux, Thinus-Blanc, \& Vauclair, 2001) conjoin geometric and nongeometric information to reorient themselves. Clearly, fish and chicks cannot rely upon linguistic processes but are nevertheless quite successful users of nongeometric features.

Despite increasing evidence that a variety of species are able to combine geometric and non-geometric information, little is known about the specific process by which different environmental features are used and combined for the purpose of spatial reorientation. Gallistel (1990) has argued that geometric 
information is used to align or match the currently perceived environment with the stored representation of the environment in a global way. It is unclear, however, whether this is a basic process, which is then further specified by taking nongeometric features into account or whether features and geometry are encoded by a more unified process from the beginning (for a detailed discussion on different coding possibilities, see Cheng and Newcombe, 2005).

Reorientation studies with neglect patients suggest the presence of separate, independent neural systems for processing geometric and nongeometric information. Guariglia, Coriale, Cosentino, and Pizzamiglio (2000) studied the reorientation behavior of patients with right brain damage with hemispatial neglect under normal conditions and under transcutaneous electrical neural stimulation (TENS), a procedure that can temporarily reduce neglect disorders. Without TENS, neglect patients were unable to use geometric features in an all-white rectangular room, i.e., they searched randomly. In a nongeometric feature condition (red panel on one of the walls), neglect patients searched the correct corner better than chance but far from the level that has been reported for healthy adults. Most interestingly, TENS differentially affected reorientation in the geometric and non-geometric condition: TENS enabled neglect patients to use geometric information. At the same time, TENS had no effect on the incorporation of nongeometric features. This differential influence of TENS suggests that different neural mechanisms are responsible for processing geometric and non-geometric information.

In the present study, the combined use of feature and sense information was impaired when participants concurrently performed a spatial task that required large-scale directional coding. Interestingly, it seems that not just any spatial task causes impairments. Ratliff and Newcombe (submitted) did not find effects of a spatial visualization task in a large rectangular space. It might be the case that in order for a spatial task to interfere with reorientation, the task needs to utilize spatial features within the test environment, and/or it needs to involve some sort of large-scale spatial coding. Our task of continuously locating the source of a sound within the test space fulfills both criteria, whereas Ratliff and Newcombe (submitted) used an imaginary task that utilized items that were not part of the experimental room. Finding the boundary conditions for spatial shadowing effects could help specify the processes that combine nongeometric and geometric information.

In the studies that used shadowing, participants were asked to shadow throughout all parts of the reorientation task. Therefore, we do not know whether shadowing impairs the encoding or the retrieval of nongeometric information or both. Future studies should address whether spatial shadowing causes an encoding or retrieval deficit.

Our study shows that non-geometric information can be used for reorientation without recourse to linguistic processes, and suggests that making spatial judgments with regard to nongeometric features is dependent upon a spatial coding process. It seems once more that small spaces have led to misleading assumptions about spatial reorientation. Previous studies have shown 
that in contrast to small spaces, reorientation in larger spaces in which distal cues are available and participants can freely move around, is not even for small children solely dependent upon informationally encapsulated geometric information. The present study provides further evidence for the assumption that reorientation in larger spaces is flexible and independent of linguistic processes (cf. Nadel \& Hupbach, 2006).

\section{REFERENCES}

Cheng, K. (1986). A purely geometric module in the rat's spatial representation. Cognition, 23, 149-178.

Cheng, K., \& Newcombe, N. (2005). Is there a geometric module for spatial orientation? Squaring theory and evidence. Psychonomic Bulletin \& Review, 12, 1-23.

Fodor, J. (1983). The modularity of mind. Cambridge, MA: MIT Press.

Gallistel, C. R. (1990). The organization of learning. Cambridge, MA: Bradford Books/MIT Press.

Gouteux, S., Thinus-Blanc, C., \& Vauclair, J. (2001). Rhesus monkeys use geometric and non-geometric information during a reorientation task. Journal of Experimental Psychology: General, 130, 505-519.

Guariglia, C., Coriale, G., \& Cosentino, T. (2000). TENS modulates spatial reorientation in neglect patients. Neuroreport, 11(9), 1945-1948.

Hermer, L., \& Spelke, E. S. (1994). A geometric process for spatial reorientation in young children. Nature, 370, 57-59.

Hermer, L., \& Spelke, E. S. (1996). Modularity and development: A case of spatial reorientation. Cognition, 61, 195-232.

Hermer-Vazquez, L., Moffet, A., \& Munkholm, P. (2001). Language, space, and the development of cognitive flexibility in humans: The case of two spatial memory tasks. Cognition, 79, 263-281.

Hermer-Vazquez, L., Spelke, E. S., \& Katsnelson, A. S. (1999). Sources of flexibility in human cognition: Dual-task studies of space and language. Cognitive Psychology, 39, 3-36.

Hupbach, A., \& Nadel, L. (2005). Reorientation in a rhombic environment: No evidence for an encapsulated geometric module. Cognitive Development, 20(2), 279-302.

Kelly, D., Spetch, M., \& Heth, C. D. (1998). Pigeons' (Columba livia) encoding of geometric and featural properties of a spatial environment. Journal of Comparative Psychology, 112, 259-269.

Learmonth, A. E., Nadel, L. \& Newcombe, N. S. (2002). Children's use of landmarks: Implication for modularity theory. Psychological Science, 13, 337-341.

Learmonth, A. E., Newcombe, N. S. \& Huttenlocher, J. (2001). Toddlers' use of metric information and landmarks to reorient. Journal of Experimental Child Psychology, 80, 225-244. 
Nadel, L., \& Hupbach, A. (2006). Cross-species comparisons in development: The case of the spatial "module." In M. H. Johnson \& Y. Munakata (Eds), Attention and performance XXI (pp. 499-511). Oxford: University Press.

Newcombe, N. S. (2005). Evidence for and against a geometric module: The roles of language and action. In J. Rieser, J. Lockman, \& C. Nelson (Eds.), Action as an organizer of learning and development, Minnesota symposium on child psychology, Volume 33 (pp. 221-241). Mahwah, NJ: Lawrence Erlbaum Associates.

Ratliff, K. R., \& Newcombe, N. S. (submitted). Is language necessary for human spatial reorientation? Reconsidering evidence from dual task paradigms. Paper in review.

Sovrano, V. A., Bisazza, A., \& Vallortigara, G. (2002). Modularity and spatial reorientation in a simple mind: Encoding of geometric and nongeometric properties of a spatial environment by fish. Cognition, 85, B51-B59.

Sovrano, V. A., Bisazza, A., \& Vallortigara, G. (2003). Modularity as a fish (Xenotoca eiseni) views it: Conjoining geometric and nongeometric information for spatial reorientation. Journal of Experimental Psychology: Animal Behavior Processes, 29, 199-210.

Vallortigara, G., Zanforlin, M., \& Pasti, G. (1990). Geometric modules in animals' spatial representations: A test with chicks (Gallus gallus). Journal of Comparative Psychology, 104, 248-254.

Zugaro, M. B., Arleo, A., Dejean, C., Burguiere, E., Khamassi, M., \& Wiener, S. I. (2004). Rat anterodorsal thalamic head direction neurons depend upon dynamic visual signals to select anchoring landmark cues. European Journal of Neuroscience, 20, 530-536. 